\title{
ISCEV standard for clinical pattern electroretinography (PERG): 2012 update
}

\author{
Michael Bach • Mitchell G. Brigell • Marko Hawlina • \\ Graham E. Holder • Mary A. Johnson • Daphne L. McCulloch • \\ Thomas Meigen $\cdot$ Suresh Viswanathan
}

Received: 13 September 2012/ Accepted: 24 September 2012/Published online: 17 October 2012

(C) Springer-Verlag Berlin Heidelberg 2012

\begin{abstract}
The pattern electroretinogram (PERG) is a retinal response evoked by a contrast-reversing pattern, usually a black and white checkerboard, which provides information about macular and retinal ganglion cell function. This document from the International Society for Clinical Electrophysiology of Vision (www.iscev.org) is a scheduled revision of the ISCEV PERG Standard, which updates and replaces the 2007 update and all earlier versions. The standard defines a single minimum stimulus and recording protocol for clinical PERG testing to assist
\end{abstract}

This study is conducted for the International Society for Clinical Electrophysiology of Vision.

M. Bach $(\bowtie)$

University Eye Hospital, Killianstr. 5, 79106 Freiburg, Germany

e-mail: michael.bach@uni-freiburg.de

M. G. Brigell

Translational Medicine, Novartis Institutes for

Biomedical Research, Cambridge, MA, USA

M. Hawlina

University Eye Hospital, Medical Centre Ljubljana,

Ljubljana, Slovenia

G. E. Holder

Moorfields Eye Hospital, London, UK

G. E. Holder

Institute of Ophthalmology, University College London, London, UK practitioners in obtaining good quality responses and to facilitate inter-laboratory comparison. The present revision tightens stimulus specifications, expands on steady-state PERG recording, addresses visual stimulus display distinctions (CRT vs. LCD), and provides a more explicit definition of response components.

Keywords Clinical electrophysiology · Electroretinogram · Pattern electroretinogram . Retina $\cdot$ Ganglion cells

\footnotetext{
M. A. Johnson

Department of Ophthalmology and Visual Sciences, University of Maryland, Baltimore, MD, USA

D. L. McCulloch

Vision Sciences, Glasgow Caledonian University,

Glasgow, UK

T. Meigen

Universitäts-Augenklinik, Würzburg, Germany

S. Viswanathan

School of Optometry, Indiana University, Bloomington,

IN, USA
} 


\section{Introduction}

The pattern electroretinogram (PERG) is a retinal biopotential evoked by a temporally modulated patterned stimulus (e.g. checkerboard or grating) of constant mean luminance. The standard PERG is recorded to abrupt contrast reversal of a black and white checkerboard pattern with central fixation. The PERG arises largely in the ganglion cells, driven by the photoreceptors and corresponding retinal cells. Since the PERG (in contrast to the flash ERG) is a local response from the area covered by the retinal stimulus image, it can be used as a sensitive indicator of dysfunction within the macular region and it reflects the integrity of the optics, photoreceptors, bipolar cells and retinal ganglion cells. Clinically, PERGs can be used in patients with abnormal pattern VEPs to establish if a central retinal disorder is present and thus differentiate between retinal and optic nerve dysfunction as a cause for the VEP abnormality. It can also be used to detect and monitor dysfunction of retinal ganglion cells caused by conditions such as glaucoma, optic neuropathies and primary ganglion cell diseases. Thus, the PERG has clinical value in both neurological and ophthalmological practice.

PERGs are small signals, typically around $2-8 \mu \mathrm{V}$ across a normal population, making PERG recording more technically demanding than standard flash ERGs. Therefore, obtaining reliable results requires careful attention to technique, including stimulus and electrode quality as well as to sources of extraneous noise. This document is intended to guide new or existing users to appropriate techniques and technological specifications required for recording a standard clinical PERG and presumes that the reader has some background knowledge of clinical electrophysiology techniques. It is further assumed that the electrophysiologic laboratory has modern equipment for recording and analysis. Although much of the document will apply equally to adults and children, the standard is not necessarily appropriate to paediatric applications.

The International Society for Clinical Electrophysiology of Vision (ISCEV) initially published "PERG Guidelines" [1]. These were consolidated into a PERG standard [2, 3], which is updated regularly. The present document is a scheduled revision superseding the 2007 update [3]. Major revisions include narrowing the stimulus specifications, expanding on steady-state recording, addressing visual stimulus display distinctions (CRT vs. LCD) and providing a more explicit definition of response components.

The ISCEV standard PERG represents a minimum protocol for recording a PERG with procedures that should allow reproducible responses to be recorded under well-defined conditions. As a minimum, it is intended that the standard method and responses be widely used, but in addition to, rather than to the exclusion of, other paradigms. Common additional PERG techniques are also briefly described in this document. Experienced users are encouraged to use additional stimuli and testing protocols to optimize testing for specific clinical applications. ISCEV has also published standards for recording the multifocal ERGs [4], full-field ERG [5], electrooculograms [6], visual evoked potential [7] and guidelines for calibration of electrophysiologic equipment [8].

\section{Waveform nomenclature and measurement}

The waveform of the PERG evoked by contrast reversal of pattern stimuli depends on the temporal frequency of the stimulus (transient versus steady-state). By convention, in the PERG waveform positivity at the active corneal electrode is displayed upward.

\section{Transient PERG}

The ISCEV standard PERG is a transient response, that is, a response that is effectively complete before the next contrast reversal. Transient recording allows separation of the PERG components. At low temporal frequencies ( $<6$ reversals per second (rps); equivalent to $<3 \mathrm{~Hz}$ ), transient PERGs are obtained (Fig. 1). The PERG waveform in normal subjects usually consists of a small initial negative component with a peak time of approximately $35 \mathrm{~ms}$ (N35), followed at 45-60 ms by a much larger positive component (P50). This positive component is followed by a large negative component at 90-100 ms (N95).

The amplitudes of the standard PERG components are normally measured between peaks and troughs. The P50 amplitude is measured from the trough of $\mathrm{N} 35$ to the peak of P50. The N95 amplitude is measured from the peak of P50 to the trough of N95. It should be recognized that measured in this way, N95 amplitude includes the P50 amplitude and P50 that of $\mathrm{N} 35$. In cases where the N35 is poorly defined, the P50 


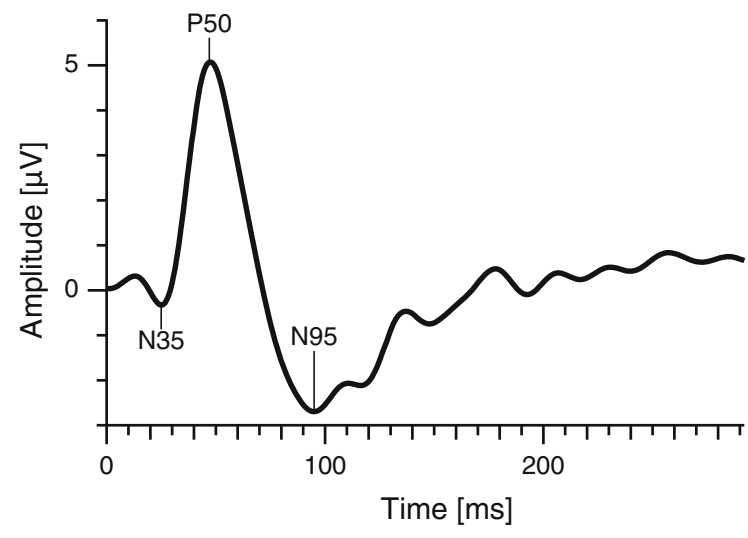

Fig. 1 A typical standard PERG. The amplitude of the P50 is typically between 2.0 and $8.0 \mu \mathrm{V}$

amplitude is measured from the average baseline (between time zero and the onset of P50) to its peak.

The time to peak (implicit time) should be measured from the onset of the contrast reversal to the peak of the component of interest. It should be noted that the highest absolute amplitude point on a waveform will not always be appropriate for the definition of the peak if there is contamination from muscle activity or other artefacts. The peak should be designated where it would appear on a smoothed or idealized waveform (see Fig. 1). The term "latency" is commonly misused when referring to the peak time or implicit time. Latency should be used to refer to the time to the onset of a response, not to the peak of the response; thus "peak time" is the preferred term.

\section{Basic technology}

Standard equipment for visual stimulus generation, amplification of physiological signals, and the recording and storing of electrophysiologic data is required for PERG testing. Information about the calibration of equipment and measurement of the parameters specified in this standard appears in the ISCEV Calibration Guidelines [8].

\section{Electrodes}

\section{Recording electrodes}

Clinical ERG electrodes which contact the cornea or nearby bulbar conjunctiva should be used as active electrodes. Electrodes that degrade image quality on the retina (this includes all contact lens electrodes) must not be used. Thin conductive fibres and foils can usually be positioned without topical anaesthesia. Electrode integrity should be checked prior to insertion, to meet manufacturer's guidelines for each electrode type. It is recommended not to measure impedance in situ unless explicitly specified by the particular equipment manufacturer. Electrodes should be carefully positioned to minimize instability (a major source of artefact or interference). Those who perform the test should be aware of possible causes of artefact.

- Fibre electrodes make contact with the globe and are best positioned in close proximity to the upper margin of the lower eyelid. This can be accomplished by placing a thin fibre electrode in the lower conjunctival fornix (under the lower eyelid), which may reduce trial-to-trial variability but will also result in lower amplitude PERG; any chosen placement must be consistent across normals and patients. Optimum stability is achieved by tethering the electrode near the nasal canthus.

- Foil electrodes should be positioned directly under the centre of the pupil so that there is minimal or no movement of the electrode when the patient blinks. This is best achieved by having the foil curve over the lower eyelashes without contacting them and then tethering the lead to the cheek. The junction of the electrode and lead should form as straight a line as possible, and this junction should not touch the skin.

- Loop electrodes should be hooked into the lower fornix. Loops should be folded so that the contact windows on the otherwise insulated wire are positioned on the bulbar conjunctiva, about $5 \mathrm{~mm}$ below the limbus. Loop electrodes should not touch the cornea. To achieve this, the limbs of the loop should diverge widely $(15-20 \mathrm{~mm}$ ) before entering the fornix. The lead is then taped to the cheek.

The appropriate placement techniques for individual electrode types are very important to achieve stable and reproducible PERG recordings.

Skin (surface) active electrodes should not routinely be used for recording the standard PERG, because skin electrodes positioned on the lower eyelid will record PERGs of lower amplitudes than those recorded from an electrode in contact with the eye. 
Skin electrode recordings may, however, be useful when a corneal electrode is contraindicated or in paediatric practice. The use of a skin electrode to record the PERG deviates from the standard and should be noted in the report.

\section{Reference electrodes}

Separate surface reference electrodes should be placed on the skin near the ipsilateral outer canthus of each eye. Mastoid, earlobe or forehead locations may result in contamination of the PERG from cortical potentials or from responses of the fellow eye. An electrode in the occluded eye may be used as a reference during monocular PERG recording.

\section{Ground electrodes}

A separate surface electrode should be attached and connected to the amplifier "ground input". The forehead would be a typical location, but other locations are acceptable. The location of the ground electrode does not affect the standard PERG.

\section{Surface electrodes}

The skin should be prepared with a suitable cleaning agent and a suitable conductive paste used to ensure good electrical connection. The impedance between the skin electrodes used for reference and ground should be less than $5 \mathrm{k} \Omega$, measured in situ. Since the electrode in the eye will have very low impedance, a low impedance of the reference electrode is also important to obtain recordings as free as possible from mains (line frequency) interference.

\section{Electrode cleaning and sterilization}

Electrodes (if not disposable) must be suitably cleaned and sterilized according to manufacturers' recommendations and current national standards for devices that contact skin and tears.

\section{Stimulus parameters}

This standard specifies the protocol for basic clinical PERG recording.

\section{Field and check size}

The stimulus for the standard PERG is a black and white reversing checkerboard. The width of the individual checks (check size) for the standard PERG is $0.8^{\circ}\left( \pm 0.2^{\circ}\right)$, and the checks should be square ( $\pm 5 \%$ error). It is not necessary to use a square stimulus field, but the aspect ratio between the width and the height of the stimulus field should be between $4: 3$ and $1: 1$. The mean of the width and the height of the stimulus field should be $15^{\circ}\left( \pm 3^{\circ}\right)$.

\section{Luminance}

The PERG is difficult to record with low stimulus luminance. A photopic luminance for the white areas of greater than $80 \mathrm{~cd} / \mathrm{m}^{2}$ is required. The mean luminance of the stimulus screen must be constant during checkerboard reversals (i.e. no transient luminance change). This is easily achieved with classical CRT (cathode-ray tube) stimulators. Note that nearly all current liquid crystal display (LCD) stimulators present a brief luminance artefact during pattern reversal, rendering them unsuitable for PERG recording unless special precautions are taken.

\section{Contrast}

The contrast between black and white squares should be maximal (close to $100 \%$ ) for the standard PERG and not less than $80 \%$. The contrast and mean luminance used should be included in reports.

\section{Visual display units (VDUs) for stimulation}

The technology of VDUs used to present pattern stimuli may affect stimulus definition and timing. The frame rate of the VDU is a significant stimulus parameter for PERGs. For raster-based CRTs, a frequency of $75 \mathrm{~Hz}$ or greater should be used. Most current LCDs present a static, non-flickering image, typically refreshed at $60 \mathrm{~Hz}$. Rarely used but possible are projection systems, plasma displays and displays using OLEDs (organic light-emitting diodes) to create the image. LCD-based displays and projectors may have a significant delay between signal input and stimulus output. This delay should be taken into account when defining time zero of the PERG. The manufacturer should provide details about the delay of 
the display device; otherwise, it is possible to measure the delay with a photodiode.

Unfortunately, frame rate or refresh rate confounds PERG peak time, because "time zero" is conventionally defined at the time where refresh begins at the top of the screen. A better time reference would be to define time zero at the time where the centre of the screen is updated. This is not widely used at this time and shortens all peak times by at least $5 \mathrm{~ms}$. Therefore, it is suggested that recordings be reported with time zero at the beginning of the screen refresh; a note should be added to reports using a different choice for time zero or for widely deviating frame rates.

\section{Background illumination}

The luminance of the background beyond the checkerboard field is not critical when using the standard PERG technique, providing dim or ordinary room lighting is used. Ambient lighting should be the same for all recordings. Care should be taken to keep bright lights out of the subjects' direct view.

\section{Reversal rate}

The standard transient PERG should be obtained using a reversal rate of $4.0 \pm 0.8$ reversals per second (rps) (corresponding to $2.0 \pm 0.4 \mathrm{~Hz}$ ). Reversal rate must be reported in rps, not in $\mathrm{Hz}$.

\section{Calibration}

All stimulus parameters including luminance and contrast should be calibrated either locally or by the manufacturer. Regular recalibration is advised [8].

\section{Recording equipment}

\section{Amplification systems}

AC-coupled amplifiers with a minimum input impedance of $10 \mathrm{M} \Omega$ are required. Amplification systems must be electrically isolated from the patient according to the current safety standards for medical recording systems. The recording frequency band of bandpass amplifiers should include the range from 1 to
$100 \mathrm{~Hz}$. Notch filters (that suppress signals at the mains line frequency) are contraindicated, as they may reduce or distort the signal. Some users may encounter severe electromagnetic interference from the stimulus display that makes it difficult to obtain satisfactory recordings with these filter settings. Ideally, such interference should be eliminated by shielding or by modifying the equipment. Rearranging the electrode leads may also be of benefit.

\section{Averaging and signal analysis}

Signal averaging is necessary because of the small amplitude of the PERG. The analysis period (sweep time) for the standard PERGs should be $150 \mathrm{~ms}$ or greater; with a 4 rps stimulation rate, the full $250 \mathrm{~ms}$ between reversals is recommended.

\section{Artefact rejection}

Computerized artefact rejection is essential. The limits for rejection should be set at no higher than $\pm 100 \mu \mathrm{V}$. The amplifiers must return to baseline rapidly following signals containing artefacts to avoid inadvertent storage of non-physiological data.

\section{Sampling rate}

A minimum sampling rate of $1,000 \mathrm{~Hz}(1 \mathrm{~ms}$ per point) is recommended. See the calibration standard [8] for further information.

\section{Data display system}

Display systems must have adequate resolution to represent accurately the characteristics of this small amplitude signal. Ideally, the recording system provides simultaneous display of the input signal and the accumulating average. In the absence of a simultaneous display, a rapid alternation between displaying the input signal and displaying the current average is advisable, so that the quality of the input signal can be adequately monitored. Even with a computerized artefact rejection system, it is important that the input signal be continuously monitored for baseline stability and for the absence of amplifier saturation. 


\section{Clinical protocol}

Preparation of the patient

\section{Positioning, pupils and pre-adaptation}

Patients should be as comfortable as possible. A stable head position is important. A headrest may considerably reduce artefacts, while a chin rest may increase muscle artefacts. The PERG should be recorded without dilation of the pupils to maximize retinal image quality. We advise that fluorescein angiography or fundus photography be avoided prior to PERG testing, but if these examinations have been performed, the patient should have at least 30 -min recovery time in ordinary room illumination before PERG recording. Pupil size should be recorded.

\section{Fixation}

A fixation mark in the centre of the screen at a node of the checkerboard is essential. If unable to see the fixation mark, patients should fixate the centre of the screen. If there is any doubt about the quality of fixation in an individual patient, one effective method is to ask the patient to point at the middle of the screen with a (laser) pointer throughout the test. Excessive blinking during recording should be discouraged; pauses in recording may be advantageous.

\section{Refraction}

Because of the nature of the stimulus, optimal image quality is necessary for PERG recordings. Patients can wear the appropriate optical correction for the test distance, or trial lenses can be used; bifocals or progressive glasses will not insure optimal imaging over the full stimulus field. The near optimal correction is particularly important for patients with reduced accommodation (i.e. presbyopia), especially for smaller displays, which will require a short viewing distance to achieve a 15-degree stimulus field.

\section{Monocular and binocular recording}

Proper positioning of recording and reference electrodes will permit either monocular or binocular recording of the standard PERG. Binocular recording is recommended for the PERG because it is generally more stable, reduces examination time and allows fixation by the better eye in cases of asymmetric visual loss. Monocular stimulation is required to record the PERG and the VEP simultaneously and in patients with ocular misalignment (e.g. strabismus).

\section{Recording}

A minimum of 100 artefact-free sweeps should be collected and averaged for a standard PERG. More sweeps, perhaps as many as 300 , will be needed when the PERG is small or undetectable or in conditions with high background noise or substantial artefacts. At least two trials for each stimulus condition should be obtained to confirm reproducibility (i.e. at least one replication). It may be beneficial to superimpose repeated PERG recordings to evaluate quality and reproducibility.

\section{PERG reporting}

\section{Reporting}

It is recommended that all reports contain measurements of P50 and N95 amplitude (see above), and P50 peak time (the peak of N95 is often rather broad precluding accurate peak time measurement of this component). All reports should also contain the stimulus parameters (luminance, contrast and field size) and normal ranges for the laboratory concerned. Pupil size should be noted. The report of PERG results should include the recorded waveforms with appropriate amplitude and time calibrations, marks for the N35, P50 and N95 components, and should show replications.

\section{Normative data/reference ranges}

There are no standard international reference ranges for PERG measurements. Each laboratory needs to establish normal values for its own equipment and patient population. It should be noted that there are PERG changes with age; over the range of $18-55$ years of age, the changes are relatively small. 


\section{Additional tests}

\section{Large field PERGs}

For some applications, users should consider recording the PERG to a larger field, such as $30^{\circ}$, in addition to the standard field size. This larger field PERG can be used in conjunction with the standard field to allow an assessment both of central and paracentral macular function. Further, a large field PERG may have an improved signal-to-noise ratio, which would be useful in patients with very abnormal responses or when noise levels are very high.

\section{Steady-state PERG}

At higher temporal frequencies, that is, above $10 \mathrm{rps}$ $(5 \mathrm{~Hz})$, the successive waveforms overlap and a "steady-state" PERG is evoked. There are situations in which the steady-state PERG is useful, in particular for glaucoma studies. Since little extra time is required, laboratories may wish to consider recording steadystate PERGs in addition to the standard transient PERG.

For steady-state PERG, a reversal rate of approximately $16 \mathrm{rps}(8 \mathrm{~Hz}) \pm 20 \%$ is recommended. Interpretation of steady-state PERGs requires measurement of amplitude and phase shift (relative to the stimulus) of the response at the reversal rate (the second harmonic) using Fourier analysis. The presence of a significant fundamental (first harmonic, i.e. at $8 \mathrm{~Hz}$ ) indicates technical problems. For correct interpretation, the analysis period must be an integer number of stimulus cycles; more than 6 cycles are advised for robust signal detection. Steady-state PERG recording without the capability for such analysis is not recommended.

Reporting of steady-state PERGs should include the amplitude and phase shift of the PERG at the reversal rate. If phase shift rather than peak time is reported, phase shift needs to be uniquely defined (namely whether it is increasing or decreasing with increasing peak time).

Acknowledgments This Standard forms part of series of Standards, Recommendations and Guidelines prepared by the International Society for Clinical Electrophysiology of Vision (ISCEV).

Conflict of interest None.

\section{References}

1. Marmor MF, Holder GE, Porciatti V, Trick GL, Zrenner E (1996) Guidelines for basic pattern electroretinography. Recommendations by the International Society for Clinical Electrophysiology of Vision. Doc Ophthalmol 91:291-298

2. Bach M, Hawlina M, Holder GE, Marmor MF, Meigen T, Vaegan, Miyake Y (2000) Standard for pattern electroretinography. Doc Ophthalmol 101:11-18

3. Holder GE, Brigell MG, Hawlina M, Meigen T, Vaegan, Bach M (2007) ISCEV standard for clinical pattern electroretinography -2007 update. Doc Ophthalmol 114:111-116

4. Hood DC, Bach M, Brigell M, Keating D, Kondo M, Lyons JS, Marmor MF, McCulloch DL, Palmowski-Wolfe AM (2012) ISCEV standard for clinical multifocal electroretinography (mfERG) (2011 edition). Doc Ophthalmol 124: $1-13$

5. Marmor MF, Fulton AB, Holder GE, Miyake Y, Brigell M, Bach M, International Society for Clinical Electrophysiology of Vision (2008) ISCEV Standard for full-field clinical electroretinography (2008 update). Doc Ophthalmol 118: 69-77

6. Marmor MF, Brigell MG, McCulloch DL, Westall CA, Bach M (2011) ISCEV standard for clinical electro-oculography (2010 update). Doc Ophthalmol 122:1-7

7. Odom JV, Bach M, Brigell M, Holder GE, McCulloch DL, Tormene AP, Vaegan (2010) ISCEV standard for clinical visual evoked potentials (2009 update). Doc Ophthalmol 120:111-119

8. Brigell M, Bach M, Barber C, Moskowitz A, Robson J (2003) Guidelines for calibration of stimulus and recording parameters used in clinical electrophysiology of vision. Doc Ophthalmol 107:185-193 